\title{
Carnets
}

Revue électronique d'études françaises de l'APEF

Deuxième série - 19 | 2020

Petite fabrique d'interprètes

\section{Sur Un Balcon en Forêt}

Fabrique Littéraire de Julien Gracq

\section{Alain Trouvé}

\section{(2) OpenEdition}

Journals

Édition électronique

URL : http://journals.openedition.org/carnets/11892

DOI : 10.4000/carnets.11892

ISSN : 1646-7698

Éditeur

APEF

Référence électronique

Alain Trouvé, "Sur Un Balcon en Forêt », Carnets [En ligne], Deuxième série - 19 | 2020, mis en ligne le 31 mai 2020, consulté le 23 décembre 2020. URL : http://journals.openedition.org/carnets/11892 ; DOI : https://doi.org/10.4000/carnets. 11892

Ce document a été généré automatiquement le 23 décembre 2020.

\section{(c) (i) \&)}

Carnets est mis à disposition selon les termes de la licence Creative Commons - Atribution - Pas d'utilisation commerciale 4.0 International. 


\title{
Sur Un Balcon en Forêt
}

\author{
Fabrique Littéraire de Julien Gracq
}

\section{Alain Trouvé}

Il s'agira d'entrevoir de quoi procède l'imagination romanesque dans Un balcon en forêt ${ }^{1}$, autrement dit d'en explorer l'arrière-texte (Gladieu, Pottier, Trouvé, 2013 et Cabral, Domingues, Laurel, 2016). Corollaire de l'intertexte, l'arrière-texte brasse dans le travail de la langue une matière mémorielle multiforme, du souvenir vécu aux réminiscences littéraires et artistiques, en passant par des données géographiques et historiques. L'enjeu sera notamment de saisir à l'œuvre et dans ses effets une forme originale de réalisme.

2 La réception du livre en 1958 fut marquée par la déception chez les adeptes des romans précédents : à l'onirisme qui paraissait le signe distinctif de l'écriture gracquienne, succède un ancrage plus précis dans la réalité historique et géographique. Parallèlement, on note une appréciation plus favorable chez ceux qui, comme Claude Roy, avaient été critiques vis-à-vis des premiers romans. Ces réactions signalent une modification d'accent à défaut d'une rupture franche.

3 L'œuvre de Gracq pose la double question du roman et du réalisme qui traversa tout le vingtième siècle. Le procès du roman entamé à la fin du siècle précédent fut porté à son paroxysme dans la phase surréaliste, avec la condamnation formulée par André Breton. Dans Le Manifeste de 1924, Breton reprit, comme on le sait, une critique déjà adressée par Valéry à une forme courante de roman symbolisée par l'incipit La marquise sortit à cinq heures ${ }^{2}$. Le procès du réalisme coïncide avec le refus de la séparation entre le réel et l'imaginaire : prétendant circuler librement de l'un à l'autre, le surréalisme selon le Manifeste promet une connaissance supérieure grâce à l'exploration de l'inconscient par les rêves et l'automatisme. On peut y voir une forme d'idéalisme au moins chez Breton, une sorte de "folie de la poésie » dira Gracq dans son André Breton (1948: 464), folie rêvant d'un absolu de connaissance effaçant la frontière entre le sujet écrivant et l'objet de son discours.

4 Gracq, très proche de Breton par la capacité de son écriture à faire surgir un monde onirique, s'en différencie spécialement par le recours au roman, massif dans la première partie de son œuvre, de 1938 à 1958, de Au château d'Argol à Un balcon en forêt, 
et en dépit des fluctuations d'accent qu'on vient d'évoquer. Une des pierres de touche de cette divergence entre les deux écrivains est sans doute l'invention de personnages, clefs de voûte du roman. Breton, ne s'y résout pas, refusant dans Nadja (1928) la coupure entre personne et personnage: "Je persiste à réclamer des noms, à ne m'intéresser qu'aux livres qu'on laisse battants comme des portes, et desquels on n'a pas à chercher la clé » (Breton, $1928: 650-651$ ).

5 L'enjeu de ce refus est le statut de la fiction et du roman, oscillant entre deux postulations. À un bout de la chaîne, certains théoriciens de la littérature ont évoqué, autour des travaux de Genette (1991), Cohn (2001), Montalbetti (2001), une " étanchéité » de la fiction, fondée sur une séparation ontologique entre le monde romanesque issu de l'imaginaire et le réel. À l'autre bout, la lignée surréaliste dérivant de Breton veut toujours transgresser cette frontière, mettant en pratique l'injonction nervalienne d'un « épanchement du songe dans la réalité ». Les théories de la lecture et de l'art comme jeu ont au contraire tenté d'articuler et de dialectiser ces deux pôles ${ }^{3}$.

6 Une autre dimension de l'écriture romanesque est le rapport aux lieux: Gracq associe de façon originale un savoir socialisé, lié à son métier de professeur d'histoire géographie, et une transfiguration poétique nourrie par ses lectures. Le regard de "professionnel de l'espace » contribue néanmoins à façonner les descriptions, même lorsqu'elles paraissent teintées d'onirisme.

7 Un risque de tautologie du commentaire a été signalé par Michel Murat (1991): il conduirait à répéter à propos de la genèse de l'écriture gracquienne ce que Gracq luimême en a dit. L'autoréflexivité de ses derniers textes est en effet assez frappante, de Lettrines (1967) aux Carnets du grand chemin (1992). Deux raisons peuvent néanmoins inciter à persister dans l'exploration des mécanismes de création, au-delà du discours auctorial, quels qu'en soient la perspicacité et l'intérêt. On peut d'abord s'appuyer sur ce que Todorov appelle la position d'exotopie du lecteur, qui fait que, lisant le roman, une tierce personne ne se confond jamais totalement avec l'auteur lecteur de soimême. La mise en perspective du roman dans l'œuvre est également facilitée par cette position extérieure. L'arrière-texte, attentif au décalage entre l'auteur et le tiers lecteur, est aussi le nom donné à cette exploration dédoublée.

Pour s'en approcher, on s'intéressera au traitement de l'espace, à la fabrication des personnages et aux effets de la fable romanesque dans la perspective d'un sujet individuel, puis collectif.

\section{L'espace : substrat historico-géographique et métamorphose romanesque}

9 La couverture de l'édition d'origine porte la mention « récit » et non «roman », afin, selon le commentaire de l'auteur, de signaler une proximité plus grande avec la réalité ${ }^{4}$. De nombreux traits d'écriture font néanmoins penser au roman, à commencer par le traitement de l'espace. Son élaboration croise une culture à forte coloration littéraire, un savoir-faire professionnel d'historien géographe et une enquête de terrain : en 1955, Gracq, selon son propre commentaire (1971), empreinte la ligne Charleville-Givet pour aller « voir l'Ardenne ». 


\section{Difficulté théorique}

Rappelons que les notions d'espace et de temps entrent en crise au $\mathrm{XX}^{\mathrm{e}}$ siècle. Dès lors que l'espace euclidien a éclaté sous l'effet des nouvelles théories physiques (de la relativité à la mécanique quantique), il ne semble plus être autre chose que la construction mentale que nous en élaborons à travers des langages successifs. La question de sa représentation intéresse particulièrement les géographes, les sémioticiens et les philosophes.

11 Peut-on concevoir un "hors-texte", comme hors langage ? À la frontière entre la sociocritique à qui l'on doit ce terme (Duchet, 1970) ${ }^{5}$ et la philosophie, les variations de Derrida $(1967,1987)$ et de Barthes $(1977,1980)$ sur la question en signalent le caractère problématique, l'un et l'autre ayant nié l'existence du hors-texte, puis produit des énoncés contradictoires avec cette déclaration ${ }^{6}$. Pour un géographe et pour un historien, la phrase de Derrida «Il n'y a pas de hors-texte " n'a pas de sens. Leurs disciplines présupposent un hors-texte mais leurs discours et leurs représentations cherchent par des voies propres une adéquation fonctionnelle.

Le sociologue et philosophe Henri Lefèvre distingue trois espaces: l'espace perçu, d'ordre phénoménologique, l'espace conçu par les urbanistes, les planificateurs, l'espace vécu, à travers images et symboles (Lefèvre, 1974). L'espace est ainsi le produit d'une corrélation qui elle-même amorce une comparaison.

13 Les marqueurs géographiques interviennent plus ou moins dans ce feuilleté de sens. Saul Kripke (1980 : 164) a proposé d'envisager les noms propres et en particulier le nom de lieu comme «désignateur rigide ». Dans le même esprit, Michel Arrivé précisa naguère : «Je tiens qu'en littérature, la géographie, c'est la toponymie ». (Arrivé, 2013 : 47).

Pour Bertrand Westphal, dans La Géocritique, «La représentation est la traduction d'une souche dans un dérivé » (2007: 126). Il s'agirait en ce sens de distinguer des images mentales dotées d'une valeur opérationnelle dans la sphère de la praxis (le nom de lieu, la carte : ce que Westphal nomme "réalèmes ») et des images mentales affectées d'un coefficient fictionnel par leur élaboration littéraire. Sans oublier que les figures de style, longtemps prises pour le propre du langage poétique, sont aujourd'hui reconnues et étudiées comme composante du langage de communication courant et du langage scientifique lui-même?

Le roman de Gracq offre à ce sujet un exemple très intéressant.

\section{La « maison forte » : du réalème au motif littéraire}

15 Il y eut quinze à vingt maisons fortes, devant la barrière naturelle de la Meuse ou devant les fortifications de la ligne Maginot. Ces installations étaient censées servir de sonnettes d'alarme pour avertir les défenses françaises d'infiltrations de l'ennemi. Remarquons qu'elles sont peut-être étudiées aujourd'hui grâce à la littérature, au roman de Gracq et à sa source littéraire. Le hors-série de la revue Terres ardennaises de février 2001 et intitulé « Des balcons en forêt Maisons fortes des Ardennes 1939-1940 », rédigé par Léon Watelet, sous-lieutenant de l'armée française en 1940, apporte nombre de précisions. Il s'agit d'un travail de chroniqueur à visée historique. Mais le titre choisi, par la référence à Gracq, montre que la littérature sert à repenser le réel historique. 

Jean-Louis de Rambures en 1970, Gracq souligne sa dette envers un roman d'Aragon, Les Communistes $^{8}$ : «Le déclic, je crois, a été quelques lignes d'Aragon dans Les Communistes, où il parle des maisons fortes de l'Ardenne, dont je ne connaissais pas l'existence » $(O C$, II :1191).

17 La fresque d'Aragon marque la fin du cycle romanesque Le Monde réel entrepris après la rupture avec le groupe surréaliste. L'écriture réaliste puise une partie de ses références dans l'enquête et la documentation. Aragon et Gracq comptent parmi les rares écrivains ayant abordé par le biais du roman la « drôle de guerre ».

Si la maison forte constitue un réalème, la force suggestive du motif tient à son caractère contradictoire. Du point de vue militaire, la construction se compose d'une maison à l'étage et d'un blockhaus en dessous. Sont donc associées une vie domestique presque normale et la présence larvée de la guerre ; cette dualité met en jeu les couples $\mathrm{vie/mort,} \mathrm{conscience/} \mathrm{inconscience,} \mathrm{sécurité/menace} \mathrm{:} \mathrm{cet} \mathrm{abri} \mathrm{est} \mathrm{le} \mathrm{lieu} \mathrm{où} \mathrm{la} \mathrm{moitié}$ de la garnison va périr. La partie fortifiée renvoie aussi bien aux défenses militaires qu'aux défenses psychologiques. La maison forte fonctionne comme interface de la vie sociale et de la vie psychique, du monde extérieur et du monde intérieur.

Ce motif se nourrit par ailleurs de souvenirs de lectures. La maison au milieu des bois rappelle les contes de fées auxquels le récit fait de nombreuses allusions. Le schème de la construction avec vue surplombante sur une sorte de mer végétale renvoie aussi à l'écriture antérieure de Gracq: dans Au château d'Argol, le cabinet installé en haut de la tour la plus élevée du château offre ce genre de vue (1938, $O C, \mathrm{I}: 28)$. En dépit de la rupture tonale, une continuité imaginaire relie ces deux récits. La maison dans le récit de 1958 est encore un château.

Le titre Un balcon en forêt se signale par sa prodigalité allégorique, au sens moderne de figure ouverte au lecteur (Benjamin, 1925). Selon Gracq lui-même, dans un entretien de 1968, ce titre évoque trois idées : la «vision du premier étage en encorbellement audessus d'un blockhaus", la notion de "personnage très contemplatif » et «l'atmosphère particulière des vacances, d'un moment "entre parenthèses", telle que l'engendrait l'ambiance de désœuvrement et d'attente de la "drôle de guerre" » (Un balcon, $O C, \mathrm{I}$ : 1313). D'autres filiations ont été identifiées par ses commentateurs. Bernhild Boie, directrice de l'édition Pléiade, signale cette phrase de Liberté Grande, recueil de proses poétique qui suit dans l'après-guerre les deux premiers romans de l'auteur:

Je redescendais chaque soir aux champs calmes, les mains pleines comme celui qui touche une femme, appuyant le front encore, les yeux fermés, ainsi que le cœur manque et qu'on marche en dormant, au songe odorant et au vide sous le soleil de ce village accoudé à la forêt comme un après-midi d'été au balcon de sa nuit sauvage. (1946, OC, I : 309)

21 D'autres lectures se montrent sensibles à l'étrange association de mots constitutive du titre : « un balcon en forêt » met en relation l'espace de la primitivité et celui d'une civilisation empreinte de raffinement, à laquelle renvoie le château convoqué ici par synecdoque. Michel Murat relève l'analogie entre la casemate de la maison, «bloc étanche, soudé autour de vous» (Un balcon, OC, I : 16) et l'écriture romanesque, «bloc compact de deux cent mille mots » (1991: 96).

22 Aux déterminations géographiques et historiques de la maison forte s'ajoute donc une forte surdétermination symbolique, ouvrant sur un statut double de l'espace.

Carnets, Deuxième série $-19 \mid 2020$ 


\section{Régime fictionnel de la géographie} delà de pensée, entre réflexion et rêverie. Elles peuvent être fonctionnelles : dans la phrase "le matelas belge manquait d'épaisseur ", l'image explique le rôle assigné à la Belgique dans la stratégie du commandement. Le manque d'épaisseur connote le motif de la sécurité trompeuse. Les drapeaux français et allemands placés sur la carte figurent «un cercle de mouches attendant autour du fromage qu'on en retirât la cloche ». Ici la valeur visuelle de l'image est limitée. On passe d'emblée à une vision interprétative où se mêlent l'hostilité à la guerre et sa biologisation, voire une figure du destin tragique si l'on songe aux mouches et à la pièce éponyme de Sartre, pourtant identifié, parfois, comme l'anti-Gracq. L'« énorme tâche verte et vivace de la forêt qui poussait en se morcelant des tentacules au-delà de la Meuse de Liège ", aide à visualiser ce qu'une carte donnerait à voir directement : une forêt aux contours irréguliers avec extensions dans différentes directions. Mais la métaphore connote aussi le monstre.

Le nom de lieu (localité, région), sous-ensemble des noms propres ou « désignateurs rigides ", revêt dans ce cadre une importance particulière car il a fortement à voir avec la question identitaire.

Paris réel, nous souscrivons aux analyses de Thomas Pavel (1988) décrivant le statut ontologique mixte du toponyme en régime romanesque. Gracq semble rejoindre Genette dans un Entretien avec Jean-Louis Tissier : "Dans une fiction, tout doit être fictif » $(1978$ : 1207). Mais sa pratique est tout autre. Le toponyme romanesque chez lui n'est pas invention pure, mais condensation d'éléments prélevés sur le réel. Moriarmé est un condensé de Monthermé (bourg situé à $20 \mathrm{~km}$ au nord de Charleville) et de Morialmé (village belge localisé au sud-est de Charleroi). Les Falizes, de même, procède de la réunion de Flizes, commune située à l'ouest de Sedan et du défilé de La Falizette, au nord-ouest de la ville. La condensation est un des deux mécanismes du Travail de rêve analysé par Freud. Non seulement la rêverie toponymique se souvient de la géographie, mais le dosage géographie/imaginaire évolue d'un roman à l'autre entraînant des effets différents.

compte les noms du fleuve (La Meuse), de la région Ardenne. On appréciera la différence avec le Farghestan, pays imaginaire du Rivage des Syrtes (1951).

"épinglée à la tête de son lit " par Grange (Un balcon, $O C, \mathrm{I}: 68$ ) : elle représente un modèle de réalité doté d'une fonctionnalité. Le héros y mesure en pensée «les cent kilomètres de forêt » d'Ardenne séparant la frontière allemande de la Meuse.

Cette forêt est hantée par les lectures du romancier. Gracq cite Michelet (Un balcon, OC, I : 68) pour l'expression « immense forêt de petits arbres ». Mais l'expression vient en réalité, selon Bernild Boie (Un balcon, $O C$, I : 1324), du géographe Vidal de La Blache, dont le Tableau de la géographie de la France a été lui-même repris dans l'Histoire de France tome I, de E. Lavisse, paru en 1911. Les souvenirs littéraires s'en mêlent, du merveilleux de la geste médiévale (Brocéliande) à Shakespeare (" la forêt galante de Shakespeare » : 123) ou à Rimbaud, si l'on tient compte de l'équivalence forêt/ mer dans l'écriture romanesque de Gracq. Jean Bellemin-Noël croit reconnaître dans l'emploi du 
néologisme "flottaison" un souvenir du Bateau ivre qu'il croise avec sa lecture du premier poème en prose des Illuminations, "Après le déluge » (2001: 26). Il invente le néologisme interlecture pour désigner le rapprochement lié à la subjectivité du lecteur, sans garantie textuelle objective. Nous y verrions, non contradictoirement, une des formes de l'arrière-texte, non plus auctorial mais lectoral, se nourrissant de références latentes tirées par le lecteur de sa propre culture. Parfois, évoquant la «sieste de cette armée au bois dormant ", la référence directe à Rimbaud se mêle à la forme générique du conte : « il y avait un charme trouble, passionnel, à se jeter dans ce bateau ivre qui avait jeté par-dessus bord son gouvernail, puis ses rames - le charme étrange du fil de l'eau ». (Un balcon, OC, I: 84). À d'autres moments, la référence latente nous paraît encore hanter l'imaginaire de la forêt gracquienne, comme dans ce passage évoquant la marche matinale de Grange pour rejoindre Mona, passage que nous proposons de lire dans la lumière du poème « Aube »:

Avant d'arriver aux Falizes, maintenant, il quittait la route à l'entrée de la clairière et prenait un chemin de terre qui se glissait entre la lisière des taillis et les clôtures d'épines des jardinets : rien ne lui plaisait autant, quand il était libre dès le lever, que d'éveiller Mona de bonne heure... (Un balcon, OC, I : 44)

Le traitement romanesque des lieux opère à un double niveau de la fiction: il recompose un monde de la fable parallèle au monde réel et encore lesté du poids d'authenticité de ce dernier, il se déploie dans toutes les directions de l'imaginaire grâce au travail du style à l'échelle de la phrase et dans la connivence avec d'autres textes, littéraires ou non.

\section{L'élaboration littéraire des personnages}

\section{Étayage historico-biographique}

30 Un mécanisme similaire participe de l'invention du personnel romanesque. S'y retrouvent des données issues de l'expérience de la vie militaire qui affleuraient déjà dans Un beau ténébreux, données issues de l'expérience du soldat Gracq durant la drôle de guerre. Michel Murat qualifie certaines pages de «document quasi ethnographique sur la vie militaire» (Un balcon, OC, I : 201). La figure de Grange brasse des données se rapportant au sujet biographique et au sujet lisant. Des articles de journaux lus en attendant Mona (Un balcon, OC, I : 48) avec leur contenu insignifiant, le "Supplément gratuit en couleur du Petit Ardennais » (p. 68) où se retrouvent des échos de la guerre en Finlande, se mêlent à des souvenirs de lecture affleurant lors de la permission à Chinon : l'officier un moment revenu à la vie civile y marche sur les traces de Nerval, «le Prince d'Aquitaine à la tour abolie » (Un balcon, OC, I : 77). L'affectation à la maison forte ressemble à une punition qui ne dit pas son nom. En tout cas, pour reprendre le mot du capitaine Varin venu rendre visite à Grange et à ses hommes, elle a tout d'un "piège à cons » (Un balcon, $O C, \mathrm{I}: 43$ ). Les rapports mitigés du héros avec l'état-major font écho à la situation de Gracq au début de la Guerre, fiché par la Sécurité militaire pour son appartenance au PC qu'il quitta avec le pacte germano-soviétique et muté en septembre 1939 au $137^{\mathrm{ème}}$ RI (Lorraine, Dunkerque, Boulonnais, Flandre). L'affectation à ce régiment où il y avait des pertes à combler relève d'une mesure semi-disciplinaire. Voici pour le substrat historico-biographique. 


\section{Fabrique littéraire}

31 Les réminiscences livresques interfèrent davantage dans la figure de Mona, la jeune veuve rencontrée dans la forêt. On retrouve avec ce personnage le puissant motif de la femme-enfant qui hanta l'écriture d'André Breton. Mona est fille de la forêt et des contes de fées, parente de Mélusine. Le récit La Route (1953-1956), inachevé et interrompu pour écrire Un balcon, puis repris dans La Presqu'île (1970), fonctionne pour certaines évocations comme un laboratoire du récit de 1958. La figure collective de femmes présente des similitudes troublantes avec Mona. Leur sensualité exubérante et instinctive attire les voyageurs engagés sur une route mythique :

La seule coquetterie qu'elles avaient c'était de choisir - une bouche cherchait votre bouche dans le noir avec une confiance têtue de bête douce qui essaie de lire sur le visage de son maître, et c'était soudain toute une femme chaude, dénouée comme une pluie, lourde comme une nuit défaite, qui se laissait couler entre vos doigts. ( $\mathrm{La}$ Route, OC, II : 413)

Avec Mona, Gracq reprend les schèmes de la pluie, de la nuit, de la sensualité orale mais corrige le côté dévalorisant de la bête par une amorce d'ancrage social, lui associant une servante. La matière mentale reste mythique comme l'indique plus directement $\mathrm{La}$ Route évoquant les « bacchantes inapaisées dont le désir essayait de balbutier une autre langue » (La Route : 416).

La dimension archaïque de cette représentation affleure dans la description de la Maison de Mona : " Maisonnette de mère Grand perdue au fond de la forêt » (Un balcon, $O C, \mathrm{I}: 10)$. Maison et mère sont indissociables dans l'imaginaire avec une extension à la mer par homonymie : « Il lui semblait qu'elle venait à lui par une route ouverte dans la mer».

\section{Croisements du monde extérieur et du monde intérieur}

Cette dimension symbolique de la métaphore, déjà aperçue à propos de l'espace, ouvre la possibilité d'une lecture analytique. La figuration symbolique est une des quatre composantes du Travail de rêve. Lors de la première soirée et de la première nuit passées aux Falizes, Grange croit voir dans Mona et sa servante Julia « deux démones rieuses " (Un balcon, OC, I : 34). Toutes les strates d'éros participent à des degrés divers de cette figure de femme. La merveille de la rencontre de l'Autre ramène à une expérience archaïque où le sujet tout puissant voit ses désirs immédiatement exaucés ; soit, dit en termes freudiens, au stade primitif dans lequel la fusion avec l'autre maternel prédomine tandis que n'a pas encore émergé, avec l'accès au symbolique, la reconnaissance d'un tiers et de l'autre comme personne. En ce sens, l'attachement à une situation et à un état peut être plus fort que l'attachement à une personne. Lorsque la menace se fait plus précise et que le capitaine Varin lui propose de se mettre à l'abri à l'arrière, Grange refuse sans lier ce refus à la présence de Mona. Il la fait néanmoins partir un peu plus tard mais viendra se réfugier, blessé, dans sa maison : "il se sentait blotti là comme dans un ventre » (Un balcon, OC, I : 135).

La fabulation ouvre ici sur une autre dimension du hors-texte, non plus le monde comme espace de l'action, mais une structure affective qui touche à la langue et cependant la précède dans l'histoire du sujet, les premiers rapports du petit être humain avec son environnement parental étant prélinguistiques. 


\section{Le roman comme épreuve de réalité fictive et fiction poétique}

Deux degrés de la fiction vont se mêler ici : degré fort, celui de la fable romanesque, degré plus diffus lié au jeu poétique sur les figures?

\section{Du jeu d'enfant au jeu littéraire}

37 L'« épreuve de réalité » en psychanalyse désigne le moment de la discrimination établie par le sujet entre satisfaction imaginaire (fantasmée) et satisfaction réelle différée dans la réalité. Le symbolique et le temps y jouent leur partie, essentielle. Michel Picard (1986) a avancé l'idée, en régime littéraire, d'une "épreuve de réalité fictive ». La littérature (spécialement le roman) nous aiderait à reprendre le travail jamais achevé de séparation.

La possibilité de discrimination grâce à la fiction romanesque n'est pas sans lien avec ce que dit Derrida sous une autre forme: «Tout récit fabuleux raconte, met en scène, enseigne ou donne à interpréter la différence sexuelle » (1994: 72-73).

39 À l'échelle du héros, l'histoire peut se lire selon trois phases : 1 / rencontre ravivant des émotions antérieures à la séparation, empreintes du souvenir délicieux des origines ; 2 / ébauche de séparation; 3 / retour à la régression sous la forme létale. Deux séquences font affleurer l'ambivalence de la figure féminine d'abord placée sous le signe de la totalité gratifiante. La scène de la luge associe la douceur sensuelle de la femme et la piqûre de la guêpe :

Il sentait les seins de Mona allongée tout contre lui s'écraser doucement contre son

dos [...] Quand ils se secouaient et s'asseyaient un instant sur la luge pour reprendre haleine, il la regardait un peu en dessous, la taille si mince dans son blouson étroit, avec une ombre de malaise : il pensait à ces guêpes qui savent d'instinct la piqûre qui peut paralyser. (Un balcon, OC, I:64)

Plus loin, le rêve du pendu, associé à Mona, mêle jouissance érotique et angoisse d'étranglement (Un balcon, $O C, \mathrm{I}$ : 79). Malgré l'éloignement de Mona intégré dans le déroulement de la fable, rester dans la maison forte, c'est, pour Grange, entretenir l'illusion d'une protection choisie envers et contre tout. L'image du retour final au ventre maternel marque le caractère régressif de cette quête.

41 À l'échelle du lecteur, il est possible de lire les images et le scénario fantasmatique sous-jacent grâce à trois facteurs discriminants : la forme narrative, l'Histoire et la Bibliothèque.

La forme narrative distingue deux romans à la troisième personne - Au château d'Argol, Un balcon - et deux autres à la première personne - Un beau ténébreux, Le Rivage des Syrtes. Cette nouvelle dichotomie ne correspond pas à l'inflexion du roman onirique vers le roman réaliste précédemment évoquée; elle donne au jeu littéraire plus de complexité et renvoie chaque œuvre à sa sémiologie propre. Il semble toutefois, et cela s'applique à Un balcon, que la troisième personne vienne mettre à distance un contenu affectif trop proche de soi et trop dangereusement mortifère. Le roman joue de la faille entre temps mythique et temps historique. Le dénouement volontairement suspendu remet au lecteur le soin de s'arranger de cette indécision. 
La bibliothèque en tant que la reconnaissance intertextuelle nous amène par ailleurs à circuler d'un texte à l'autre, à porter attention au symbolique, à lire les émotions et non plus seulement à les vivre par procuration. L'hétérogénéité des matériaux écrits journaux, livres d'histoire, littérature - accentue peut-être cette attention discriminante.

4 Plusieurs éléments compliquent ce mécanisme de maîtrise de l'Imaginaire par le Symbolique. Grange, lui-même présenté comme grand lecteur, de Tolstoï, Gide, Shakespeare, Swedenborg (Un balcon, OC, I : 49-50) ne semble pas trouver dans ses lectures la force suffisante pour s'arracher à la puissance létale. L'aspiration à l'unité traverse nombre des références intertextuelles qui lui sont associées, imposant la recomposition d'une grande unité rêvée où viendraient se fondre la fée de Nerval, l'Ophélie de Rimbaud (« Mona trouvée dans les bois, flottée par la mer » : 62), Mélusine (revue par le Breton d'Arcane 17, publié en 1945).

5 On pourrait évoquer ici l'écriture surréaliste en tant qu'écriture postfreudienne se posant plus ou moins en rivale de l'analyse. Tel fut le cas de Breton tentant de conjuguer et de transcender dans L'Amour fou (1937) la production des images et leur interprétation ${ }^{10}$. Gracq se contente pour sa part de mettre en résonance les images et d'en laisser l'interprétation au lecteur.

\section{Foisonnement de l'écriture poétique ; arrière-texte et « pro-texte »}

Ricœur (1965) avait souligné la fécondité et les limites de la théorie freudienne de l'art comme sublimation. Car la sublimation, conversion de pulsion en buts élevés, ne supposerait comme moteur de la créativité que les forces obscures renvoyant au passé de chaque individu.

4 Un autre moteur pourrait être ce qui s'invente à travers les mots dans le dialogue de l'individu avec la cité en tant que l'humanité crée elle-même son propre avenir. Le grand roman selon Gracq serait "élation vers l'éventuel » (1980: 621). Remarquons l'ambivalence du terme "élation ", qui signifie au sens ancien : "noblesse exaltée du sentiment »et au sens analytique: «exaltation provenant d'un sentiment d'autosatisfaction narcissique ${ }^{11}$ ». Ajoutons ce que dit Gracq de la dimension oraculaire du rêve (1981: 1223), mais pas au sens d'une prophétie d'événement. Il s'agirait plutôt d'une quête de connaissance dépassant l'horizon de l'époque conjuguée à une dimension métaphysique hantée par l'horizon de la mort. Gracq est, rappelons-le, un pseudonyme choisi par l'écrivain Louis Poirier pour sa consonnance avec le mot «Graal ». Le poète se pense en Perceval moderne. Le Roi pêcheur (1948) est une variation théâtrale sur ce mythe et c'est encore une épigraphe empruntée au Parsifal de Wagner qui ouvre le roman de 1958. Wagner ou le rêve de l'art total comme accès à un absolu de sens.

48 Contre cette tentation, Gracq joue sur le double registre de la fable à lire et du grimoire des images à interpréter : "Failles profondes qui séparent les pages d'un livre » (Un balcon, $O C, \mathrm{I}: 59)$. On peut intégrer dans cette interprétation élargie par le lecteur la remarque sur l'abandon de la scène de la messe de minuit dans la rédaction définitive du roman :

Toute la première partie du Balcon en forêt a été écrite dans la perspective d'une messe de minuit aux Falizes, qui devait être un chapitre très important, et qui aurait 
donné au livre, avec l'introduction de cette tonalité religieuse, une assiette tout

autres. (1967: 152)

49 l'angle individuel. Au château d'Argol et Un beau ténébreux marquent la connexion entre la veine surréaliste et le romantisme, grâce à l'attention portée par l'écriture à un audelà de l'expérience ordinaire. Cet au-delà est traité sous l'angle collectif dans Le Rivage des Syrtes, sans doute influencé par le roman de Ernst Jünger, Sur les falaises de marbre $(1939)^{12}$. Sous un titre général à visée allégorique Le Rivage laisse entrevoir l'ambition de dégager par l'écriture une poésie de l'Histoire.

Ici la fable romanesque rejoint l'Histoire en un point plus précis, restituant quelque chose de la conscience collective en ce moment qui précède l'invasion de juin 1940. Cette conscience, dépassée par un réel devenu ininterprétable, en proie au songe et au primat de la sensation, s'abandonne à une sorte de régression collective. La perception des militaires dans les chars apparaît déconnectée du réel : « un camarade lui avait parlé un jour de « la sécurité étrange, irraisonnée qu'on trouvait brusquement rien qu'à rouler ainsi, le bourrelet du casque appuyé au blindage, dans le tintamarre énorme " (Un balcon, OC, I : 37). Plus loin le nuage soulevé par le convoi est comparé à « un lourd cocon de poussière ». Le rêve contamine la chose militaire : " une armée rêveuse » note Grange dans un passage en monologue intérieur ; "je rêve ici, nous rêvons tous » (Un balcon, $O C, \mathrm{I}: 87)$. La vision troublée apparaît encore dans le décalage entre les allusions historiques (les événements de Finlande, du Caucase) et le rendu par la presse locale ( $L e$ Petit Ardennais) : « il ne se passait rien » (Un balcon, OC, I : 67).

\section{Dialogue du romancier avec les historiens}

53 Le roman s'attache au retentissement dans les consciences. Un balcon, de ce point de vue, est peut-être plus intéressant en tant que contradiction non résolue que les modèles interprétatifs que Gracq déclare admirer : on pense à Spengler $\left(1981: 1215^{13}\right)$.

Grange ressemble un tout petit peu au Fabrice de Stendhal à Waterloo, assistant au spectacle de la guerre sans rien y comprendre, mais à la faillite du point de vue surplombant liée au regard de son héros, Stendhal oppose au moins par instants et 
notamment dans les premières pages de La Chartreuse des considérations à la troisième personne susceptibles de donner sens aux événements.

Et pourtant une partie de la Deuxième Guerre Mondiale s'est jouée là, dans cette forêt d'Ardenne où a eu lieu la percée des divisions blindées allemandes préparant l'encerclement de Dunkerque. La défaite et l'Occupation ont constitué le plus douloureux retour à la réalité après des mois de faux assoupissement sans que soient mis au jour tous les mécanismes de la défaite éclair.

\section{Esquisse de poétique comparée}

56 Comparons un instant cette écriture à celle des Communistes. Aragon s'est essayé à une évocation littéraire de la guerre à travers une écriture romanesque différente, opposant à l'éclairage restreint d'un point donné du dispositif la fresque politicosociale et la tentative de reconstitution à grande échelle de l'ensemble du front. Il l'a fait dans les limites de sa propre vision d'écrivain communiste, ce qui l'a amené en 1966 à une réécriture. L'écrivain attentif aussi à la diversité foisonnante du réel a cherché dans un élargissement esthétique à dépasser un horizon immédiat décidément obscurci. Je fais allusion ici aux saisissantes pages sur le désastre de Dunkerque, écrites sous le signe de Breughel, pages que l'on peut mettre en résonance, toutes proportions gardées, avec celles où Grange sent plus qu'il ne comprend la catastrophe s'abattre sur lui (Un balcon, $O C$, I : 123-124). On peut lire aussi, dans un autre registre d'écriture les pages désopilantes de Gracq sur Dunkerque dans Lettrines, évoquant des souvenirs et réflexions personnels. On pense notamment à l'épisode «La nuit des ivrognes », d'où le personnage de Gourcuff a probablement été démarqué : «Le souvenir que j'ai gardé de Dunkerque est beaucoup plus fantasmagorique que sinistre » (1967: 196-199). Aragon et Gracq, par des moyens dissemblables mais à partir d'un objet commun, représentent deux solutions littéraires à la transposition de la catastrophe dans l'écriture.

Selon Gracq (1980), nous sommes en retard dans la compréhension de la mécanique romanesque parce que les que les outils d'analyse restent à inventer. Nous ne sommes pas loin de partager ce point de vue.

Sans prétendre à une nouvelle hégémonie, l'outil arrière-texte vient néanmoins, en corollaire de l'intertexte, combler en partie ce manque et jeter un début de lumière sur le comment et le pourquoi du recours au roman, sur son inflexion, ici, vers un nouveau réalisme. L'intégration à l'arrière-texte de la dimension hors-texte comme hors-langage, permet d'en appréhender l'originalité. En ce sens, on pourrait avancer cet axiome : l'œuvre littéraire n'est faite que de mots, mais c'est peut-être parce qu'il existe du hors-texte (hors-langage) qu'il y a de la littérature.

59 Dans l'écriture inédite de 1958, qu'on l'appelle ou non romanesque, la perspective subjective, ancrée dans l'imaginaire et le fantasme, devient emblématique du collectif ou au moins d'une large part de ce dernier. Il ne s'agit pas, avec l'arrière-texte, de ressusciter en l'état la critique des sources ou la critique biographique après les avancées de l'intertextualité. Plutôt de saisir des articulations permettant de comprendre le seuil qualitatif entre la chose crée et les matériaux dont elle procède, selon une sorte de dialectique de l'arrière-plan et du plan projeté dans la pratique verbale.

60 On est frappé chez Gracq par un souci de cohérence et de maîtrise sans cesse menacé par un débordement de l'imaginaire. Comme Breton dans Le Manifeste et dans L'Amour 
fou, Gracq a rêvé d'une écriture totale qui jouerait de la contradiction entre le réel et l'imaginaire pour la dépasser dans une unité supérieure, d'ordre poétique, dont la surréalité serait une version. Son écriture littéraire et spécialement romanesque, travaillée par l'hétérogénéité, s'ouvre en contrepoint à éclatement baroque né de l'attention au réel, du jeu de certaines images, de la grande navigation symbolique dans les textes, appréhendés comme ensemble partiellement hétérogène.

Cette hétérogénéité se lit au plan littéraire selon les deux degrés de la fiction, celui de la figure, domaine de la parole poétique, celui de la fable, donc du roman. Si dans le cadre de la fable, le jeu consiste à s'adonner à la logique narrative ouverte par principe aux interprétations du lecteur, dans l'écriture poétique c'est le savoir socialisé de l'historien et du géographe qui vient interagir comme un second garde-fou avec le répertoire des figures et avec les dérives de l'imaginaire qui lui sont associées.

\section{BIBLIOGRAPHIE}

ARRIVÉ, Michel (2013). « Toponymie et littérature », in GLADIEU, M.-M., POTTIER, J.-M., TROUVE, A. (dir.), Approches Interdisciplinaires de la Lecture, $\mathrm{n}^{\circ} 7$, « Les Référents du littéraire ». Reims : Épure, pp. 47-61.

BARTHES, Roland (1977). « Leçon inaugurale au Collège de France » (2002). Cuvres complètes. V, Paris : Le Seuil.

BARTHES, Roland (1978) 4 décembre. « Des mots pour faire entendre un doute », Propos recueillis par Françoise Tournier, Elle (1981). Le grain de la voix (2002). Cuvres complètes, V. Paris : Le Seuil. BARTHES, Roland (1980). La Chambre claire (2002). CEuvres complètes, V. Paris : Le Seuil.

BELLEMIN-NOËL, Jean (2001). Plaisirs de vampire. Paris : PUF.

BENJAMIN, Walter (1925). « Allégorie et Trauerspiel », in Origine du drame baroque allemand, trad. Sibylle Muller. Paris : Flammarion coll. «Champs».

BRETON, André (1928). Nadja (1988). CEuvres complètes, I. Paris : Gallimard, « La Pléiade, dir. Marguerite Bonnet.

COHN, Dorrit (2001). Le Propre de la fiction. Paris : Le Seuil.

DERRIDA, Jacques (1967). De la Grammatologie. Paris : Le Seuil.

DERRIDA, Jacques (1987). « Où commence et comment finit un corps enseignant ? ", conférence prononcée le 14 mars 1987 lors d'un colloque organisé à Paris par le Collège international de philosophie.

DERRIDA, Jacques (1994). « Fourmis », in Lectures de la différence sexuelle, textes réunis et présentés par Mara Negron. Paris : Des femmes.

GENETTE, Gérard (1991). Fiction et Diction. Paris : Le Seuil. 
GLADIEU, Marie-Madeleine, POTTIER, Jean-Michel, TROUVÉ, Alain (2013). L'Arrière-texte Pour repenser le littéraire. Bruxelles, Peter Lang. (2016). O Arrière-texte Para Repensar o literário. CABRAL, Maria de Jesus, DOMINGUES, João, LAUREL, Maria Hermínia, Tradução e apresentação, Edições pedago. GRACQ, Julien $(1989,1995)$. Euvres complètes, I et II. Paris : Gallimard, « La Pléiade ». GRACQ, Julien (1938). Au Château d'Argol. Paris, Corti. 1989. Cuuvres complètes, I. Paris : Gallimard, «La Pléiade».

GRACQ, Julien (1945). Un Beau Ténébreux. Paris : Corti (1989). Cuvres complètes, I. Paris : Gallimard, «La Pléiade ».

GRACQ, Julien (1946). Liberté grande. Paris : Corti (1989). Cuvres complètes, I. Paris : Gallimard, « La Pléiade ».

GRACQ, Julien (1948). André Breton. Paris : Corti (1989). CEuvres complètes, I, Paris : Gallimard, « La Pléiade ».

GRACQ, Julien (1951). Le Rivage des Syrtes. Paris : Corti (1989). Cuvres complètes, I. Paris : Gallimard, «La Pléiade ».

GRACQ, Julien (1967, 1974). Lettrines, Lettrines 2. Paris : Corti (1995). Cuvres complètes, II. Paris : Gallimard, « La Pléiade ».

GRACQ, Julien (1970). Entretien avec Jean-Louis de Rambures, Le Monde, repris dans Carnets du grand chemin (1995). CEuvres complètes, II. Paris : Gallimard, « La Pléiade ».

GRACQ, Julien $(1971,1972)$. « Sur Un balcon en forêt », entretien de Julien Gracq avec G. Ernst, repris dans L'Herne, Julien Gracq.

GRACQ, Julien (1978). « Entretien avec Jean Louis Tissier » (1995). CEuvres complètes, II. Paris : Gallimard, « La Pléiade ».

GRACQ, Julien (1980). En Lisant en Écrivant. Paris : Corti (1995). Cuuvres complètes, II, Paris : Gallimard, «La Pléiade».

GRACQ, Julien (1981). « Entretien avec Jean Roudaut » (1995). Cuvres complètes, II. Paris : Gallimard, « La Pléiade ».

GRACQ, Julien (1992). Carnets du grand chemin (1995). Cuuvres complètes, II. Paris : Gallimard, « La Pléiade ».

KRIPKE, Saul $(1980,1982)$. La Logique des noms propres. Paris : Minuit.

LEFÈVRE, Henri (1974). La Production de l'espace. Paris : Anthropos.

MONTALBETTI, Christine (2001). « Fiction, réel, référence », Littérature, $\mathrm{n}^{\circ}$ 123, décembre.

MURAT, Michel (1991). Julien Gracq. Paris : Belfond.

PAVEL, Thomas (1988). Univers de la fiction. Paris : Le Seuil.

PICARD, Michel (1986). La Lecture comme jeu. Paris : Minuit.

RICEUR, Paul (1965). De l'Interprétation. Paris : Le Seuil.

SCHAEFFER, Jean-Marie (1999). Pourquoi la fiction? Paris : Le Seuil.

SoUNAC, Frédéric (2010). « Mélophobie "critique" et modèle musical dans L’Homme sans qualités de Robert Musil ", in GLADIEU, M.-M. et TROUVE, A. (dir.), Approches Interdisciplinaires de la Lecture, $\mathrm{n}^{\circ} 4$, «Voir et entendre par le roman ». Reims : Épure, pp. 143-161. 
TROUVE, Alain (2004). Le Roman de la lecture. Liège: Mardaga.

WINNICOTT, Donald W. $(1971,1975)$. Jeu et réalité. Trad. Claude Monod et Jean-Bertrand Pontalis.

Paris : Gallimard, « Connaissance de l'inconscient ».

\section{NOTES}

1. Julien Gracq, Un balcon en forêt, Paris, Corti, 1958 ; rééd. Euvres complètes (OC), II, Paris, Gallimard, « La Pléiade », édition Bernhild Boie, 1995.

2. Dans En lisant, en écrivant (OC II : 636), Gracq argumente contre Breton et Valéry à partir de cet incipit devenu emblème également de l'arbitraire de l'écriture romanesque à quoi il oppose la cohérence de cette dernière. Dans son essai consacré à l'auteur de Nadja, il y revient, précisant que la cible de Breton est le roman réaliste naturaliste (« Battant comme une porte », 1948, OC, I, : 446-472).

3. Michel Picard, dans La Lecture comme jeu (1986), s'appuie notamment sur les travaux du psychanalyste Winnicott traitant de l'art comme objet transitionnel (Winnicott, 1971) ; Jean-Marie Schaeffer dans Pourquoi la fiction? (1999), met l'accent, selon une perspective philosophique aristotélicienne, sur le pouvoir de modélisation de la fiction qui dialogue avec le monde réel mais s'en distingue par le jeu.

4. Commentaire de l'auteur dans Arts, 17-23 septembre 1958, repris dans CEuvres complètes, II, 1280.

5. La notion de hors-texte est introduite au début des années 1970 en sociocritique par Claude Duchet dans le $\mathrm{n}^{\circ} 1$ de la revue Littérature. Mais l'acception donnée à ce terme dans les écrits relevant de la sociocritique reste flottante.

6. Barthes : «Le langage humain est sans extérieur : c'est un huis clos » (1977). Derrida : «Il n'y a pas de hors-texte » (1967). La reconnaissance de l'existence de la chose face à l'objectif du photographe (Barthes, 1980) ou de la physique du corps en prise sur la parole (Derrida, 1987) tendent à montrer le contraire.

7. Voir à ce sujet, Barthes, encore : «Dans la mathématique, il y a une richesse d'imagination énorme, des grands modèles de pensée logique, une pensée qui arrive à se faire d'une façon très vivante, uniquement sur les formes et sans tenir compte des contenus. Tout cela intéresse au plus haut point la littérature. Et, dans la littérature, il y a de plus en plus un mouvement vers des formes de pensée mathématique. Il y a un niveau où mathématique et littérature se rejoignent » (1978 : 331-332.).

8. La fresque Les Communistes, $1^{\text {ère }}$ version, a paru en six volumes à la Bibliothèque française du 15 juillet 1949 au 27 avril 1951. Une seconde édition récrite est réalisée en 1966 pour les Euvres Romanesques Croisées (ORC) d'Elsa Triolet et Aragon (Paris, Robert Laffont, 42 volumes, 1964 à 1974). L'édition des CEuvres Romanesques Complètes d'Aragon (Paris, Gallimard, "La Pléiade », 5 volumes, 1997-2012) reprend cette seconde version. Les passages qui ont pu inspirer Gracq figurent dans le volume IV, pp. 144, 188 et 1416.

9. Nous nous permettons de renvoyer ici à notre essai de 2004 et à son chapitre « Lecture et fiction ".

10. Voir encore à ce sujet dans notre essai le chapitre «Cristal et sempervivum : l'écriture allégorique dans L'Amour fou d'André Breton » (2004 : 171-178).

11. Le Grand Robert, tome II : 1939.

12. Sur la mise en correspondance de Jünger et de Tacite, voir En lisant, en écrivant (1980, OC, II : 708-711): la forêt séparant la France de la Germanie y est dotée d'une même dimension allégorique. 
13. Gracq déclare à Jean Roudaut : «J'ai une dette envers Spengler, qui triche plus d'une fois avec les faits, mais dont la mise en écho généralisé de l'histoire m'a beaucoup séduit » (1981: 1215). Sur Spengler, on pourra lire encore Frédéric Sounac commentant le «bréviaire des illusions du sujet allemand des années 20, porté à se réconforter dans l'exaltation morbide de l'Urseelentum, "l'âme fondamentale", nécessairement musicale en terre germanique » $(2010: 151)$.

\section{RÉSUMÉS}

Cet article s'intéresse aux mécanismes de l'écriture-lecture littéraire dans Un balcon en forêt de Julien Gracq. Ce roman marque chez l'auteur une inflexion de l'écriture onirique vers le réalisme, qui accentue sa divergence avec le Manifeste surréaliste d'André Breton. Elle est favorisée par le double métier d'historien géographe et d'homme de lettres exercé par Gracq. Tout ne s'explique pas dans cette écriture par le rapport entre textes, autrement dit par l'intertextualité. Qu'il s'agisse de l'espace, des personnages ou du jeu proposé au(x) lecteur(s), l'analyse recourt à la notion d'arrière-texte, accordée au hors-texte comme "hors langage", pour en faire entendre les accents particuliers.

This article looks at the mechanisms of literary writing-reading in Julien Gracq's Un balcon en forêt. This novel marks in the author a shift from dream writing to realism, which accentuates its divergence from André Breton's Surrealist Manifesto. It is favored by Gracq's dual profession of historian-geographer and man of letters. Not everything in this writing can be explained by the relationship between texts, in other words by intertextuality. Whether it is the space, the characters, or the "playing" offered to the reader (s), the analysis resorts to the notion of arrièretexte, accorded to the hors-texte as well as the "out-of-language", in order to make its particular accents heard.

\section{INDEX}

Mots-clés : arrière-texte, intertextualité, hors-texte, surréalisme, réalisme, géographie, espace, personnage, jeu

Keywords : arrière-texte [sic], intertextuality, hors-text, surrealism, realism, geography, space, character, playing

\section{AUTEUR}

\section{ALAIN TROUVÉ}

Université de Reims

alain.trouve[at]wanadoo.fr 